\title{
Subcarrier and Power Adaptation for Multiuser OFDM-based Cognitive Radio Systems
}

\author{
Gaurav Bansal ${ }^{\dagger}$, Ziaul Hasan ${ }^{\dagger}$, Md. Jahangir Hossain*, and Vijay K. Bhargava ${ }^{\dagger}$ \\ ${ }^{\dagger}$ Electrical and Computer Engineering, The University of British Columbia, Canada \\ Email: \{gauravbs, ziaulh, vijayb\}@ece.ubc.ca \\ *INRS, Canada, Email:jahangir@ece.ubc.ca
}

\begin{abstract}
This paper investigates the subcarrier and power allocation problem for an OFDM-based multiuser cognitive radio (CR) system. For CR systems it is important to keep the interference introduced to the primary user (PU) band within a tolerable range. Since the joint subcarrier and power allocation problem is a non-convex integer problem and a closed form solution is difficult to find, a suboptimal algorithm that separates subcarrier and power allocation is proposed. First, a suboptimal subcarrier algorithm is proposed that allocates subcarriers to CR users that not only increase the capacity, but also reduces the interference introduced to the primary user (PU) band. Further, for a given subcarrier allocation an optimal power loading algorithm is proposed that maximizes the capacity of CR users while keeping the interference introduced to the PU band and the total power below a threshold. Simulation results show that for CR systems the proposed algorithm is able to load power into the subcarriers while keeping the interference below a specified threshold.
\end{abstract}

\section{INTRODUCTION}

Radio spectrum is one of the most scarce and valuable resources for wireless communications. Actual field measurements by various groups around the globe e.g., SpectrumPolicy Task Force appointed by the Federal Communications Commission (FCC), have reported that the allocated spectrum is highly underutilized, with utilization ranging from 15 to $85 \%$ [1], [2]. Spectrum efficiency can be increased significantly by giving opportunistic access of the frequency bands to a group of cognitive radio (CR) users to whom the band has not been originally allocated. CR is an emerging technology which would allow secondary users (or CR users) to smartly sense and make an efficient use of the available spectrum that has been licensed to primary users (PUs).

Due to its flexibility in allocating the spectrum, Orthogonal frequency division multiplexing (OFDM) has been recognized as a modulation technology for CR systems [3]. Since both CR user and primary user (PU) may exist in side-by-side bands yet have different access technologies, mutual interference is the limiting factor for performance of both networks. As there is mutual interference between CR user and PU when they co-exist in side-by-side bands [4], use of the classical loading algorithms e.g., uniform power but variable rate and waterfilling algorithms, for CR system may result in higher mutual interference in the PUs' band. In [5], we proposed power loading algorithms, that maximizes the downlink transmission capacity of the $\mathrm{CR}$ user while keeping the interference induced to the PUs below a specified threshold.
In our previous work [5], [6], we have developed power loading schemes for a single CR user based OFDM systems. In this paper, we extend power loading schemes to multiuser scenario. There are number of resource allocation algorithms which maximize capacity for conventional multiuser OFDM systems [7], [8], [9]. However, for CR scenario design of such algorithms is much more challenging because primary and CR users co-exist in adjacent bands. The problem formulation is to decide which CR users the subcarrier should be allocated and how much power and bits should be allocated in that subcarrier, such that the total interference introduced to the primary user band remains below a specific threshold.

The optimization of capacity maximization problem obtained by joint consideration of the channel qualities of various $\mathrm{CR}$ users as well as the distance of subcarriers from the PU band is computationally intensive. Specifically, the optimization problem is discrete and finding a closed form solution is difficult. In this paper, we follow the approach in [8], and we first propose a sub-optimal algorithm for subcarrier allocation. For, a given subcarrier allocation, we propose an optimal power loading algorithm which maximizes the capacity of CR users while keeping the interference introduced to the PU band and the total power below specified constraints.

Numerical results, demonstrate the strength of the proposed scheme. Specifically, it is shown that our proposed scheme might load a bit less capacity as compared to classical scheme [8], proposed scheme always maintain the interference introduced to the PU band below the interference threshold. Classical scheme is not suitable for OFDM-based CR systems, and does not satisfy the interference constraint.

The organization of the paper is as follows. Section II, describes the system model and the problem formulation. In Section III, we propose a sub-optimal algorithm for subcarrier allocation. The algorithm for optimal power adaptation for a given subcarrier allocation is presented in Section IV. Selected numerical results are given in Section V and finally, Section VI concludes the paper.

\section{System Model and Problem Formulation}

We consider the side-by-side CR radio access model, Fig. 1 as in [5]. It is assumed that the frequency bands of bandwidth $B_{1}, B_{2}, \ldots, B_{L}$, are occupied by the PU(s) $1,2, \ldots, L$, and are sensed by the CR system consisting of $M$ users. The unoccupied band sensed by the CR system for possible transmission 


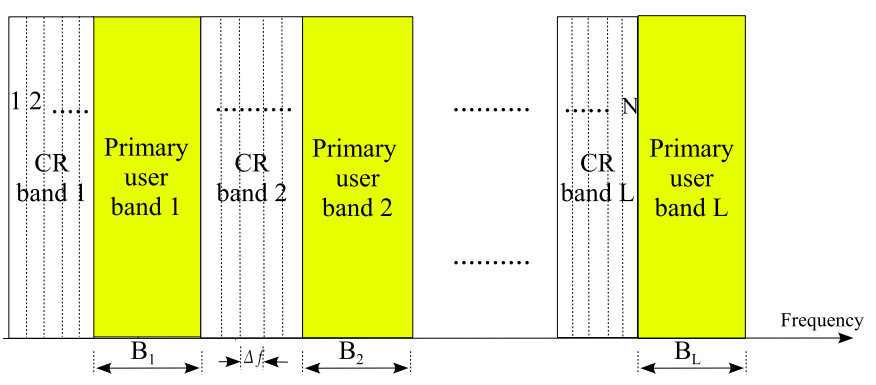

Fig. 1. Distribution of primary and CR users.

is located on each side of $L$ PU bands as shown in Fig. 1. The available bandwidth for CR transmission is divided into $N$ subcarriers. It is assumed that the bandwidth for each $\mathrm{CR}$ subcarrier is $\Delta f \mathrm{~Hz}$.

The transmission rate for the $k^{t h} \mathrm{CR}$ user for $n^{t h}$ subcarrier, for a power of $P_{k, n}$, channel gain between CR user transmitter and receiver of $h_{k, n}^{s s}$ can be expressed via Shannon capacity formula [10], [5] as,

$$
R_{k, n}=\log _{2}\left(1+\frac{\left|h_{k, n}^{s s}\right|^{2} P_{k, n}}{\sigma^{2}+\sum_{l=1}^{L} J_{k, n}^{(l)}}\right)
$$

where $\sigma^{2}$ is the AWGN noise variance and $\sum_{l=1}^{L} J_{k, n}^{(l)}$ denotes the interference introduced by all $L$ PU bands into $k^{\text {th }} \mathrm{CR}$ user's $n^{\text {th }}$ subcarrier. Here, we assume that the channel gain $h_{k, n}^{s s}$ is perfectly known at the CR transmitter.

There are two types of interference in the CR system: (1) The interference introduced by the $k^{\text {th }} \mathrm{CR}$ user transmitting in $n^{\text {th }}$ subcarrier into the $l^{\text {th }}$ PU band $\left(I_{k, n}^{(l)}\right)$. It can be written as $I_{k, n}^{(l)}=P_{k, n} * F_{k, n}^{(l)}$, where $F_{k, n}^{(l)}=$ $\left|h_{k, n}^{s p}\right|^{2} T_{s} \int_{d_{n l}-B_{l} / 2}^{d_{n l}+B_{l} / 2}\left(\frac{\sin \pi f T_{s}}{\pi f T_{s}}\right)^{2} d f$ [4], [5]. Here $T_{s}$ is the symbol duration, $h_{k, n}^{s p}$ denotes the channel gain between $k^{t h}$ $\mathrm{CR}$ user transmitter and the $l^{\text {th }} \mathrm{PU}$ receiver, and $d_{n l}$ represents the spectral distance between the $n^{\text {th }} \mathrm{CR}$ subcarrier and $l^{\text {th }} \mathrm{PU}$ band. We assume that both primary and CR users are located in a same device in a co-located scenario. Hence, primary receiver can estimate the channel gain $h_{k, n}^{s p}$ and report it to the CR transmitter [5]. Therefore, we assume that $h_{k, n}^{s p}$, and so, $F_{k, n}^{(l)}$ is known at the CR transmitter. (2) The interference introduced by the $l^{\text {th }} \mathrm{PU}$ band into $k^{\text {th }} \mathrm{CR}$ user transmitting in $n^{t h}$ subcarrier is denoted by $J_{k, n}^{(l)}$. $J_{k, n}^{(l)}$ depends on $h_{k, n}^{p s}$ which denote the channel gain between the $l^{\text {th }}$ PU transmitter and the $k^{\text {th }} \mathrm{CR}$ user receiver [5]. We assume that for colocated scenario CR transmitter can estimate $h_{k, n}^{p s}$, and so $J_{k, n}^{(l)}$ is known as in [5].

The optimization problem is to maximize the normalized capacity of the subcarriers for various CR users while keeping total interference introduced to the PU band remains below a certain threshold and total power remains below a constraint.
It can be written mathematically as follows:

$$
\max _{P_{k, n}, \rho_{k, n}} \sum_{k=1}^{K} \sum_{n=1}^{N} \rho_{k, n} \log _{2}\left(1+\frac{\left|h_{k, n}^{s s}\right|^{2} P_{k, n}}{\sigma^{2}+\sum_{l=1}^{L} J_{k, n}^{(l)}}\right)
$$

subject to,

$$
\begin{gathered}
\sum_{k=1}^{K} \sum_{n=1}^{N} \rho_{k, n} P_{k, n} F_{k, n}^{(l)} \leq I_{t h}^{(l)} \quad \text { for all } l \\
P_{k, n} \geq 0, \quad \text { for all } k, n \\
\sum_{k=1}^{K} \sum_{i=1}^{N} P_{k, n} \leq P_{T}, \\
\rho_{k, n}=\{0,1\} \quad \text { for all } k, n \\
\sum_{k=1}^{K} \rho_{k, n}=1 \text { for all } n
\end{gathered}
$$

where $P_{T}$ denotes the total power budget, $I_{t h}^{(l)}$ denotes the interference threshold for the $l^{\text {th }}$ PU band, and $\rho_{k, n}$ indicates which user is occupying a particular subcarrier. It can have only value of either 0 or 1 , as only one user can occupy a particular subcarrier.

However, to find a closed form solution for the joint subcarrier and power allocation problem is difficult because it is a discrete optimization problem. In literature [7], [8], for classical systems this problem has been solved by separately finding the subcarrier allocation and power allocation solution. Here, we first solve the subcarrier allocation problem in a sub-optimal fashion. Then for a given subcarrier allocation, we propose optimal algorithm for power allocation. In the next section, we first present the algorithm for sub-optimal subcarrier allocation.

\section{Suboptimal Subcarrier Allocation}

The algorithm is based on the algorithm in [7], [8] for classical systems. The classical algorithm allocates subcarriers according to channel-to-noise ratio (CNR), such that every user with high CNR gets more subcarriers. However in CR scenario, the interference constraint has to be taken into account. In the subcarrier allocation algorithm, equal power is assumed in all subcarriers. For all the interference constraints and power constraint to satisfy, we load equal power as follows:

$$
\begin{array}{r}
P_{e q}=\min \left\{\frac{P_{T}}{N}, \frac{I_{t h}^{(1)}}{\sum_{k=1}^{K} \sum_{n=1}^{N} F_{k, n}^{(1)}}, \frac{I_{t h}^{(2)}}{\sum_{k=1}^{K} \sum_{n=1}^{N} F_{k, n}^{(2)}},\right. \\
\left.\ldots, \frac{I_{t h}^{(L)}}{\sum_{k=1}^{K} \sum_{n=1}^{N} F_{k, n}^{(L)}}\right\}
\end{array}
$$

Further, it should be observed that subcarriers should be allocated to users with low $\sum_{l=1}^{L} F_{k, n}^{(l)}$, as it will allocate less interference to the PU band. We propose a statistic as follows,

$$
S_{k, n}=\frac{\left|h_{k, n}\right|^{2}}{\left(\sigma^{2}+\sum_{l=1}^{L} J_{k, n}^{(l)}\right) * \sum_{l=1}^{L} F_{k, n}^{(l)}}
$$


Subcarriers are allocated to various CR users according to $S_{k, n}$. By using $S_{k, n}$ instead of CNR, we are assigning subcarrier's such that not only the capacity can be maximized but also the interference introduced to the PU band can be maintained below a threshold. We define $\Omega_{k}$ as the set of subcarriers assigned to user $k$. The proposed algorithm is as follows:

1) Initialization

a) Set $R_{k, n}=0, A=\{1,2, \ldots, N\}$ and $\Omega_{k}=\phi$ for $k=$ $1,2, . ., K$

2) For $k=1$ to $K$

a) find $n$ satisfying $S_{k, n} \geq S_{k, j}$ for all $j \in A$

b) assign $\Omega_{k}=\Omega_{k} \bigcup\{n\}, A=A-\{n\}$ and update $R_{k, n}$ according to Eq. (1).

3) While $A \neq \phi$,

a) find $k$ satisfying $\sum_{n=1}^{N} R_{k, n} \leq \sum_{n=1}^{N} R_{i, n}$ for all $i, 1 \leq$ $i \leq K$

b) for the found $k$, find $n$ satisfying $S_{k, n} \geq S_{k, j}$ for all $j \in$ A

c) for the found $k$ and $n$, assign $\Omega_{k}=\Omega_{k} \bigcup\{n\}, A=$ $A-\{n\}$ and update $R_{k, n}$ according to Eq. (1).

The subcarrier allocation is done in a fashion such that the CR users which introduce low interference to the PU band and increase the capacity, has been given more subcarriers. The algorithm is suboptimal in a sense that equal power has been assumed in all subcarriers, however the complexity of the algorithm is low. Now, in the next section we propose an optimal scheme power allocation for a given subcarrier assignment.

\section{Optimal Power Distribution For A GiVEN SubCARRIER ALLOCATION}

To a certain determined subchannel allocation, the optimization power allocation problem is formulated as

$$
\max _{P_{k, n}} \sum_{k=1}^{K} \sum_{n \in \Omega_{k}} \log _{2}\left(1+\frac{\left|h_{k, n}^{s s}\right|^{2} P_{k, n}}{\sigma^{2}+\sum_{l=1}^{L} J_{k, n}^{(l)}}\right)
$$

subject to,

$$
\begin{gathered}
\sum_{k=1}^{K} \sum_{n \in \Omega_{k}} P_{k, n} F_{k, n}^{(l)} \leq I_{t h}^{(l)} \text { for all } l \\
P_{k, n \geq 0, \quad}^{\quad \text { for all } k, \text { and } n \in \Omega_{k}} \\
\sum_{k=1}^{K} \sum_{n \in \Omega_{k}} P_{k, n} \leq P_{T},
\end{gathered}
$$

where $\Omega_{k}$ are disjoint for all $k$ and $\Omega_{1} \cup \Omega_{2} \cup \ldots \cup \Omega_{K} \subseteq$ $\{1,2, \ldots, N\}$.

Theorem 1: The maximization of total transmission capacity in Eq. (5), given the constraints in Eqs. (6), (7), and (8) is given by

$$
P_{k, n}^{*}=\left[w_{k, n}-\frac{\sigma^{2}+\sum_{l=1}^{L} J_{k, n}^{(l)}}{\left|h_{k, n}^{s s}\right|^{2}}\right]^{+} \quad \text { for all } k, \text { and } n \in \Omega_{k},
$$

where $w_{k, n}=\frac{1}{\beta+\sum_{l=1}^{L} \lambda_{l} F_{k, n}^{(l)}}$ and $\beta$, and $\lambda_{l}$ are values which are determined by a method described in the proof.

Proof: We use the fact that maximization of a concave function in Eq. (5) is equivalent to minimization of its negative value. We introduce the Lagrange multipliers $\lambda_{l}$ for the inequality constraint in Eq. (6), $\mu_{i}$ for the inequality constraint in Eq. (7), and $\beta$ for the inequality constraint in Eq. (8). We can write the Karush-Kuhn-Tucker (KKT) conditions as [11]

$$
\begin{aligned}
& P_{k, n} \geq 0, \\
& \text { for all } k \text {, and } n \in \Omega_{k} \\
& \sum_{k=1}^{K} \sum_{n \in \Omega_{k}} P_{k, n}-P_{T} \leq 0, \\
& \sum_{k=1}^{K} \sum_{n \in \Omega_{k}} P_{k, n} F_{k, n}^{(l)}-I_{t h}^{(l)} \leq 0, \\
& \text { for all } l \\
& \mu_{k, n} \geq 0 \text {, } \\
& \text { for } \quad \text { all } k \text {, and } n \in \Omega_{k} \\
& \mu_{k, n} P_{k, n}=0 \text {, } \\
& \text { for all } k \text {, and } n \in \Omega_{k} \\
& \begin{array}{rl}
\beta & \geq 0, \\
\beta\left(\sum_{k=1}^{K} \sum_{n \in \Omega_{k}} P_{k, n}-P_{T}\right)^{2} & 0, \\
\lambda_{l} & \geq 0,
\end{array} \\
& \lambda_{l}\left(\sum_{k=1}^{K} \sum_{n \in \Omega_{k}} P_{k, n} F_{k, n}^{(l)}-I_{t h}^{(l)}\right)=0, \\
& \text { for all } l \\
& \begin{aligned}
-\frac{1}{\left(\frac{\sigma^{2}+\sum_{l=1}^{L} J_{k, n}^{(l)}}{\left|h_{k, n}^{s s}\right|^{2}}+P_{k, n}\right)}-\mu_{k, n} & +\beta+\sum_{l=1}^{L} \lambda_{l} F_{k, n}^{(l)} \\
& =0,
\end{aligned}
\end{aligned}
$$$$
\text { for } \quad \text { all } k \text {, and } n \in \Omega_{k}
$$

Now, we can eliminate $\mu_{k, n}$ from Eq. (10) and write the equation as follows,

$$
\begin{aligned}
P_{k, n} & \geq 0, \\
& \text { for } \quad \text { all } k, \text { and } n \in \Omega_{k} \\
\sum_{k=1}^{K} \sum_{n \in \Omega_{k}} P_{k, n}-P_{T} & \leq 0, \\
\sum_{k=1}^{K} \sum_{n \in \Omega_{k}} P_{k, n} F_{k, n}^{(l)}-I_{t h}^{(l)} & \leq 0, \\
\frac{1}{\left(\frac{\sigma^{2}+\sum_{l=1}^{L} J_{k, n}^{(l)}}{\left|h_{i}^{s s}\right|^{2}}+P_{k, n}\right)} & \text { for all } l \\
& \leq \beta+\sum_{l=1}^{L} \lambda_{l} F_{k, n}^{(l)},
\end{aligned}
$$




$$
\begin{aligned}
& \begin{aligned}
& \text { for } \quad \text { all } k \text {, and } n \in \\
& P_{k, n} \beta-\frac{P_{k, n}}{\left(\frac{\sigma^{2}+\sum_{l=1}^{L} J_{k, n}^{(l)}}{\left|h_{k, n}^{s s}\right|^{2}}+P_{k, n}\right)}+P_{k, n} \sum_{l=1}^{L} \lambda_{l} F_{k, n}^{(l)}
\end{aligned} \\
& =0 \text {, } \\
& \text { for } \quad \text { all } k \text {, and } n \in \Omega_{k} \\
& \beta \geq 0 \text {, } \\
& \beta\left(\sum_{k=1}^{K} \sum_{n \in \Omega_{k}} P_{k, n}-P_{T}\right)=0, \\
& \lambda_{l} \geq 0, \\
& \text { for all } l \\
& \lambda_{l}\left(\sum_{k=1}^{K} \sum_{n \in \Omega_{k}} P_{k, n} F_{k, n}^{(l)}-I_{t h}^{(l)}\right)=0, \\
& \text { for all } l
\end{aligned}
$$$$
\text { If } \beta+\sum_{l=1}^{L} \lambda_{l} F_{k, n}^{(l)}<\frac{1}{\left(\frac{\sigma^{2}+\sum_{l=1}^{L} J_{k, n}^{(l)}}{\left|h_{k, n}^{s s}\right|^{2}}\right)} \text {, then condition } 4 \text { in }
$$

Eq. (11) can only hold if $P_{k, n}^{*}>0$, which by solving condition

5 in Eq. (11) gives, $P_{k, n}^{*}=\frac{1}{\beta+\sum_{l=1}^{L} \lambda_{l} F_{k, n}^{(l)}}-\frac{\sigma^{2}+\sum_{l=1}^{L} J_{k, n}^{(l)}}{\left|h_{k, n}^{s s}\right|^{2}}$.

On the other hand, if $\beta+\sum_{l=1}^{L} \lambda_{l} F_{k, n}^{(l)} \geq \frac{1}{\left(\frac{\sigma^{2}+\sum_{l=1}^{L} J_{k, n}^{(l)}}{\left|h_{k, n}^{s s}\right|^{2}}\right)}$

then $P_{k, n}^{*}>0$ is impossible, because it would violate the condition 5 in Eq. (11). Therefore, we can write the optimal power profile as

$$
\begin{aligned}
P_{k, n}^{*}= & \frac{1}{\beta+\sum_{l=1}^{L} \lambda_{l} F_{k, n}^{(l)}}-\frac{\sigma^{2}+\sum_{l=1}^{L} J_{k, n}^{(l)}}{\left|h_{k, n}^{s s}\right|^{2}} \\
& \text { if } \beta+\sum_{l=1}^{L} \lambda_{l} F_{k, n}^{(l)}<\frac{1}{\left(\frac{\sigma^{2}+\sum_{l=1}^{L} J_{k, n}^{(l)}}{\left|h_{k, n}^{s s}\right|^{2}}\right)} \\
= & 0 \text { if } \beta+\sum_{l=1}^{L} \lambda_{l} F_{k, n}^{(l)} \geq \frac{1}{\left(\frac{\sigma^{2}+\sum_{l=1}^{L} J_{k, n}^{(l)}}{\left|h_{k, n}^{s s}\right|^{2}}\right)}(12)
\end{aligned}
$$

which is equivalent to

$P_{k, n}^{*}=\left[w_{k, n}-\frac{\sigma^{2}+\sum_{l=1}^{L} J_{k, n}^{(l)}}{\left|h_{k, n}^{s s}\right|^{2}}\right]^{+} \quad$ for all $k$, and $n \in \Omega_{k}$,

where $w_{k, n}=\frac{1}{\beta+\sum_{l=1}^{L} \lambda_{l} F_{k, n}^{(l)}}$.

As $P_{k, n}$ is constrained by condition 2 in Eq. (11), it implies $\frac{1}{\left(\frac{\sigma^{2}+\sum_{l=1}^{L} J_{k, n}^{(l)}}{\left|h_{k, n}^{s s}\right|^{2}}+P_{k, n}\right)}>0$. Therefore, $\beta$ and $\lambda_{l}$ cannot be all zero simultaneously as it would violate the condition 4 in Eq. (11).

We introduce $\gamma_{j}$, and $c_{j}, \forall j=\{1,2, . . L, L+1\}$, s.t. $\gamma_{j}=\lambda_{j} \forall j=\{1, . . L\}$, and $\gamma_{L+1}=\beta$, and $c_{j}=F_{k, n}^{(j)} \forall j=$
$\{1, . . L\}$, and $c_{L+1}=1$. We can write $w_{k, n}=\frac{1}{\sum_{j=1}^{L+1} c_{j} \gamma_{j}}$. We define two sets $A$ and $B$ such that if (i) $\gamma_{j}>0$, then $j \in A$ and if (ii) $\gamma_{j}=0$, then $j \in B$. As $\beta$ and $\lambda_{l}$ cannot be all zero simultaneously, $A$ would be a non-empty set.

To calculate $\gamma_{j}$ we first assume $\gamma_{j}>0, \forall j=\{1, . . L, L+$ $1\}$, i.e. $B$ to be an empty set and solve the following $(L+1)$ equations corresponding to $(L+1) \gamma_{j}$ constraints,

$$
\begin{aligned}
\sum_{k=1}^{K} \sum_{n \in \Omega_{k}} P_{k, n} F_{k, n}^{(l)}-I_{t h}^{(l)} & =0, \quad \text { for all } l, \\
\sum_{k=1}^{K} \sum_{n \in \Omega_{k}} P_{k, n}-P_{T} & =0 .
\end{aligned}
$$

Now, if solution to above equations exists, and corresponding $P_{k, n}^{*}$ values satisfy conditions 1,2 , and 3 in Eq. (11) then $P_{k, n}^{*}$ is optimal. If not, we assume $\gamma_{j}=0$ for exactly one $j \in\{1, . . L, L+1\}$, i.e. we assume the cardinality of set $B$ to be $1(|B|=1)$. We solve, corresponding $L$ equations for $\gamma_{j}$ constraints, and see if solution exist and satisfy the conditions 1,2 , and 3 in Eq. (11). It should be noted that $|B|$ can be 1 in $(L+1)$ ways (by considering all $(L+1) \gamma_{j}$ 's to be zero exactly once) and all cases would be taken into account. If the solution is still not found, we keep on repeating the process until $|B|=L$, and $|A|=1$. Due to the strict concavity of the objective function there will be a unique solution. This completes the proof of the Theorem.

\section{Numerical Results}

We assume the values of $L, N$, and $K$ to be 4,20 , and 5 respectively. The values of $T_{s}, \Delta f, B_{1}, B_{2}, B_{3}$, and $B_{4}$ have been assigned to $4 \mu$ seconds, $0.3125 \mathrm{MHz}, 1 \mathrm{MHz}, 2 \mathrm{MHz}, 5$ $\mathrm{MHz}$, and $10 \mathrm{MHz}$ respectively. Noise variance $\left(\sigma^{2}\right)$ has been taken to $10^{-6}$ and the channel gain $h_{k, n}^{s s}, h_{k, n}^{s p}$, and $h_{k, n}^{p s}$ have been assumed to be Raylegih Fading with an average channel power gain equal to 1 . The values of $I_{t h}^{(l)} \forall l=1,2, . . L$, have been assumed to be $2 \times 10^{-6} \mathrm{~W}$.

In Fig. 2, we plot the achievable normalized capacity for the CR users versus total power budget for the proposed scheme and the classical scheme. Here, by classical scheme we mean the scheme described in [8]. It should be noted that for the classical scheme in our simulations, we don't cosider the fairness constraint given in [8]. As, classical scheme does not have the interference constraint, we observe in Fig. 2 that classical scheme is able to load more capacity in CR user band as compared to proposed scheme. However from Fig. 3, we can observe classical scheme is not able to maintain the interference threshold, i.e. it will introduce more interference to the PU band and hence, is not suitable for OFDM-based CR systems. Our proposed scheme is able to load power into multi-CR users giving increased capacity while always keeping the interference introduced to the PU band below a specified threshold. 


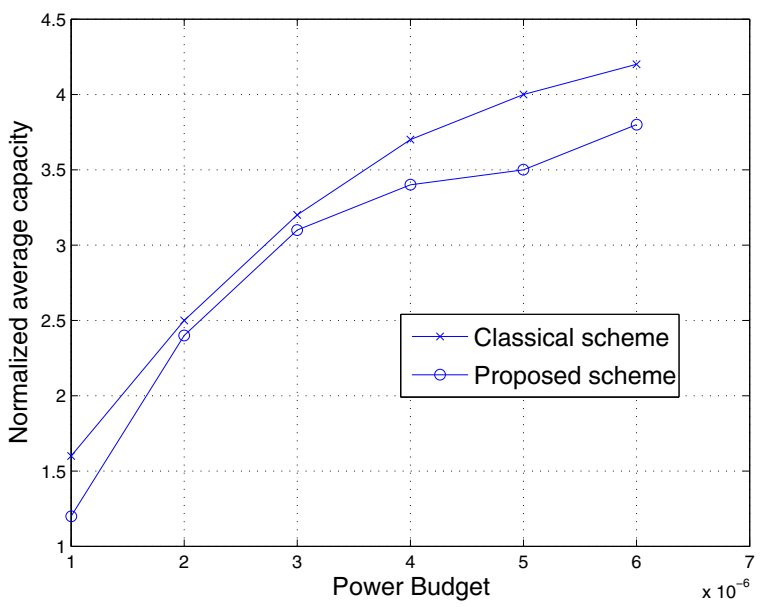

Fig. 2. Power Budget $P_{\text {total }}$ vs. normalized capacity for the CR users.

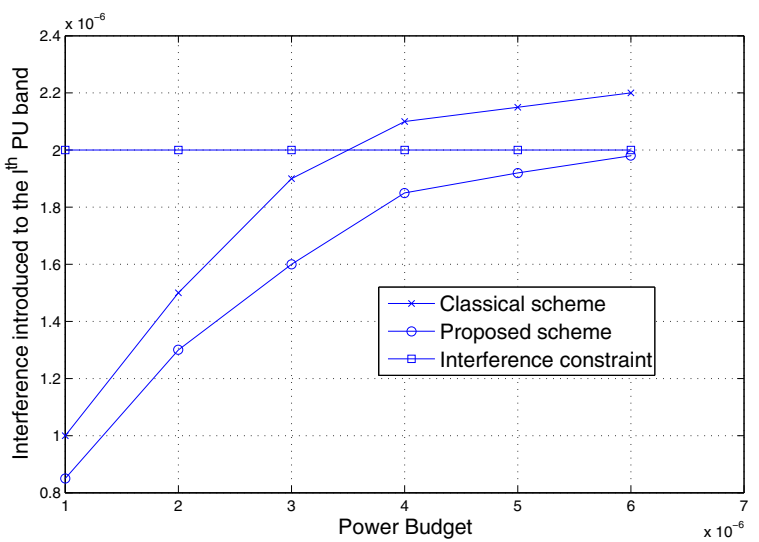

Fig. 3. Power Budget $P_{\text {total }}$ vs. interference generated to $l^{\text {th }} \mathrm{PU}$ band.

\section{CONCLUSION}

In this paper, we considered the problem of subcarrier and power allocation for multiuser OFDM-based CR systems. We proposed a suboptimal subcarrier allocation algorithm that allocates subcarriers to CR users in a manner such that not only the capacity of CR users is increased but also the interference introduced to the PU band is minimized. Further, we proposed an optimal power allocation algorithm for a given subcarrier allocation. The proposed algorithm maximizes the capacity of CR users while simultaneously maintaining the interference and power constraints. Presented simulation results show that the proposed scheme load increased capacity into $\mathrm{CR}$ users while always maintaining the interference and power constraints.

\section{ACKNOWLEDGEMENT}

This research was supported by the Natural Sciences and Engineering Research Council of Canada under a Canada Graduate Scholarship and in part under a Strategic Project Grant.

\section{REFERENCES}

[1] S. M. Mishra et al., "A real time cognitive radio testbed for physical and link layer experiments," in Proc. of IEEE Int. Symposium on Dynamic Spectrum Access Networks (DySPAN’ 05), pp. 562-567, Nov. 2005.

[2] Federal Communications Commission, "Spectrum Policy Task Force," Rep. ET Docket no. 02-135, Nov. 2002.

[3] T. Weiss, and F. K. Jondral, "Spectrum pooling: an innovative strategy for the enhancement of spectrum efficiency," IEEE Commun. Mag., vol 43, no. 3, pp. S8-S14, Mar. 2004.

[4] T. Weiss, J. Hillenbrand, A. Krohn, and F. K. Jondral, "Mutual interference in OFDM-based spectrum pooling systems," in Proc. of IEEE Vehicular Technol. Conf. (VTC'04), vol. 4, pp. 1873-1877, May 2004.

[5] G. Bansal, Md. J. Hossain, and V. K. Bhargava, "Optimal and suboptimal power allocation schemes for OFDM-based cognitive radio systems," in IEEE Trans. on Wireless Commun., vol. 7, No. 11, pp. 4710-4718, Nov. 2008.

[6] G. Bansal, Md. J. Hossain, and V. K. Bhargava, "Link Adaptation in OFDM-based cognitive radio systems," in Cognitive Radio Communications Networks, Springer-Verlag, pp. 189-211, 2007.

[7] W. Rhee, and J. M. Cioffi, "Increase in capacity of multiuser OFDM system using dynamic subchannel allocation," in Proc. of IEEE Vehicular Technol. Conf. (VTC'00), vol. 2, pp. 1085-1089, May 2000.

[8] Z. Shen, J. G. Andrews, and B. L. Evans, "Adaptive resource allocation in multiuser OFDM systems with proportional rate constraints, " in IEEE Trans. on Wireless Commun., vol. 4, No. 6, pp. 2726-2737, Nov. 2005.

[9] C. Y. Wong, R. S. Cheng, K. B. Letaief, and R. D. Murch, "Multiuser OFDM with adaptive subcarrier, bit, and power allocation," in IEEE Journal on Selected Areas in Commun., vol. 17, No. 10, pp. 1747-1758, Oct. 1999.

[10] T. M. Cover, and J. A. Thomas, Elements of Information Theory, John Wiley and Sons, 1991.

[11] S. Boyd, and L. Vandenberghe, Convex Optimization, Cambridge University Press, 2004. 